945 ELDERLY WOMEN'S EXPERIENCES OF SELF-SAMPLING FOR HPV TESTING

R Hermansson*, M Olovsson, C Gustavsson, A Lindstrom. Uppsala University, Uppsala, Sweden

\subsection{6/ijgc-2021-ESG0.559}

Introduction/Background* Self-sampling for Human Papillomavirus (HPV) testing, as an alternative to the conventional speculum based sampling, is highly acceptable to women of screening ages. The aim of this study was to describe older women's (60 to 75 years) experiences of self-sampling.

Methodology In Sweden a descriptive study with quantitative and qualitative methods was designed to collect data from a survey of women who participated in self-sampling for HPV testing at home. Individual interviews were done with women who tested positive in the first self-sampling, and were either negative in their second HPV test or were positive in their second HPV test, but without precancerous lesions or cancer.

Result(s)* Of eligible women, 97.2\% answered the survey. Among the surveyed women, $49.2 \%$ reported it was very easy to perform self-sampling, $46.8 \%$ answered it was easy and $2.0 \%$ answered it was not easy. A majority (58.9\%) answered that they prefer self-sampling, $16.5 \%$ that they prefer sample collection by a healthcare provider, $23.7 \%$ did not have any preference and $0.9 \%$ did not answer the question. In the interviews, 13 of 16 invited women participated. Most of them reported that they prefer self-sampling because it was easy to perform, less embarrassing and less time consuming than a visit to a clinic. The majority of women reported that they were not worried when informed about having an HPV positive test. Participating women with better knowledge about the significance of an HPV infection were more worried about having a positive HPV test.

Conclusion* Cervical cancer remains a highly preventable disease through screening and early treatment. Our results indicated that vaginal self-sampling for HPV testing was a wellaccepted method for cervical cancer prevention in this group of older women.

\section{EFFICACY OF HPV GENOTYPING AND SIMULTANEOUS CYTOLOGICAL EVALUATION IN CENTRAL BLACK SEA REGION OF TURKEY}

1,2 B Tatar*. 'Samsun Training and Research Hospital, Gynecologic Oncology, Samsun, Turkey; ${ }^{2}$ Dr Burak Tatar Clinic, Samsun, Turkey

10.1136/ijgc-2021-ESGO.560

Introduction/Background* The distribution of human papillomavirus (HPV) varies geographically, and each country is making its own screening and vaccination program. This study questioned the need for colposcopy for HPV types other than HPV 16 and 18, and the need for cytology incorporated into HPV testing.

Methodology 1043 consecutive patients referred from August 2017 to November 2019 for colposcopy are included in the study. For statistical analysis, logistic regression analysis, ANOVA and Pearson's correlation was used. The value of $p$ $<0.05$ was considered statistically significant.

Result(s)* HPV 16 was the most common HPV type referred, followed by HPV 18, 52, 51 and 31, respectively. HPV 16 tends to be positive in younger patients than other HPV types ( $p<0.05)$. For all HPV positive patients with cytological
Abstract 976 Table 1 Biopsy results of all cytological abnormalities regardless of HPV status.

\begin{tabular}{|c|c|c|c|c|c|}
\hline & \multicolumn{3}{|c|}{ Normal and CIN 1} & \multicolumn{2}{|c|}{ CIN 2+ } \\
\hline & (n) & $\%$ & & (n) & $\%$ \\
\hline ASC- & 170 & & $73,0 \%$ & 63 & $27,0 \%$ \\
\hline \multicolumn{6}{|l|}{ US } \\
\hline LSIL & 115 & & $74,2 \%$ & 40 & $25,8 \%$ \\
\hline AGC & 11 & & $78,6 \%$ & 3 & $21,4 \%$ \\
\hline ASC-H & 19 & & $63,3 \%$ & 11 & $36,7 \%$ \\
\hline HSIL & 14 & & $51,9 \%$ & 13 & $48,1 \%$ \\
\hline
\end{tabular}

Abstract 976 Table 2 Biopsy results of patients with cytological abnormality and with hrHPV positivity other than HPV 16 or 18 $(n=120)(p>0.05$ for all parameters).

\begin{tabular}{|c|c|c|c|c|c|c|}
\hline & \multicolumn{2}{|c|}{$\begin{array}{l}\text { Normal and } \\
\text { CIN } 1\end{array}$} & \multicolumn{3}{|c|}{ CIN 2+ } & \multirow[b]{2}{*}{$\begin{array}{l}\text { Lower - Upper for } 95 \% \\
\mathrm{Cl}\end{array}$} \\
\hline & (n) & $\%$ & (n) & $\%$ & $\mathrm{OR}$ & \\
\hline ASC- & 43 & $71,6 \%$ & 17 & $28,3 \%$ & 1,012 & $0,5-2,02$ \\
\hline US & & & & & & $0,38-1,82$ \\
\hline LSIL & 34 & $75,5 \%$ & 11 & $24,5 \%$ & 0,83 & $0,09-8,49$ \\
\hline AGC & 3 & $75,0 \%$ & 1 & $25,0 \%$ & 0,85 & $0,49-13,32$ \\
\hline ASC-H & 3 & $50 \%$ & 3 & $50 \%$ & 2,66 & $0,6-24.0$ \\
\hline HSIL & 2 & $40 \%$ & 3 & $60 \%$ & 3,83 & \\
\hline
\end{tabular}

abnormality, only HSIL cytology increased the risk of CIN $2+$ lesions (OR:5.7, 1.1-29.6 95\% CI) $(\mathrm{p}<0.05)$. 19\% of the CIN $2+$ lesions were detected in patients without HPV 16 and 18 infection (cytology and double other high-risk HPV positivity). Only HPV 16 (OR: 1.25, 0.9-2.2 95\% CI) and HPV 33 (OR:2.76; 1,18-6.49 95\% CI) $(\mathrm{p}<0.05)$ had prediction for CIN $2+$ lesions. In patients with only a cytological abnormality or double other hr HPV positivity but without HPV 16 and 18 infection, we detected 159 (19\%) CIN 2+ lesions.

Conclusion* HPV 33 may be implemented in high-risk HPV screening protocols for direct colposcopy referral among HPV 16 and HPV 18 in specific regions. If we had been opted HPV-based screening for only HPV 16 and 18 without cytology, $19 \%$ of all CIN $2+$ lesions would have been missed. HPV based screening only with HPV 16 and 18 does not seem to be feasible. Nonavalent vaccines may be considered for vaccination for this specific sub-population.

\section{CAN GENETICS ANALYSIS OF KARYOTYPE HELP US TO PREDICT CANCER IN ANY PATIENTS BEFORE ANY SYMPTOMS? LET'S SEE OUR OBSERVATION IN OUR CASES}

${ }^{1}$ V Krsic* ${ }^{1}$ B Jocic Pivac, ${ }^{2}$ J Krsic. ${ }^{1}$ GAK Narodni Front, Obgyn, Belgrade, Serbia; ${ }^{2}$ Military Academy of Belgrade, Chirurgico, Belgrade, Serbia

\subsection{6/ijgc-2021-ESG0.561}

Introduction/Background* One in 1000 to one in 1500 pregnancies are complicated by cancer. We would like to show 
three cases in our institution how we discover cancer in pregnancy, although patients did not have any symptoms before pregnancy.

Methodology CASES 1: 29-year- old women, healthy women in 10 weeks gestation did Prenatal test, Livia, and they could not do analysis because they could not extract fetal blood. All her laboratory and pregnancy were in normal limit.

CASE 2: 33-year-old pregnant women in 10 weeks of gestations did Prenatal test, Veracity, and laboratory could not extract fetal blood. She was healthy with previous laparoscopic operation due endometriosis on the left side on ovaria.

CASE 3: 42-year-old pregnant women in 10 weeks of gestation did Prenatal test, NIFTY, and laboratory could not extract fetal blood. In previous history she had operation of breast cancer and hemotherapy 4 years ago.

Result(s)* CASE 1 Patient at 36 weeks of gestation patient had big palpable mass on site liver and we performed, Caesarean Section and discovered carcinoma of colon sigmoid with multiple metastasis in abdomen. The newborn was in good condition and she lived one year later.

CASE 2 Patient at 33 weeks of gestation patient had pain in her legs. Detail examination showed changes on all bones suspected on metastatic changes. We performed Caesarean Section, and we found Krukenber's tumor with multiple metastasis in abdomen. The newborn was healthy and she lived for one month later.

CASE 3 Patient at 16 weeks of gestation patient had pain in abdomen. We did an open laparotomy and found colon cancer with meta changes in abdomen. Caesarean Section was performed in 33 weeks of gestation. Two newborns were healthy and she is alive.

Conclusion* Patients were healthy before pregnancy and Prenatal tests showed large changes in their karyotypes in terms of multiple mitoses. At the time of obtaining the results, neither the patients nor the doctors suspected they have cancer.

Our conclusion is that changes in the karyotype, as in these cases, could be the first indicator that there is cancer in the body. Could analysis of karyotype single out the risk population and be gold standard to look for cancer before the onset of symptoms?

\section{THE DEVELOPMENT OF POLICIES AND PREVENTIVE MEASURES FOR CANCER IN GENERAL AND GYNECOLOGICAL CANCERS BASED IN REPORTING METHODS: A TRANSVERSAL STUDY}

${ }^{1} \mathrm{~F}$ Kamberi*, ${ }^{2} \mathrm{E}$ Sinaj. "University of Vlore "Ismail Qemali", Vlore, Faculty of Health, Research Centre of Public Health, Vlora, Albania; ${ }^{2}$ University of Medicine Tirana, Faculty of Technical Medical Science, Albania

\subsection{6/ijgc-2021-ESG0.562}

Introduction/Background* In Albania, cancers including gynecological cancers are the leading causes of death among the adult population.

Methodology The purpose was assessing how much easier the participants, healthy women in a cross-sectional study reported whether family or someone close to them has cancer.

Result(s)* $17 \%$ of 1012 women participants randomly selected in various institutions in Vlore, Albania reported that a close family was diagnosed with cancer. Close friends, friends were affected respectively $13.44 \%$ and $19.24 \%$, while $6 \%$ prefer not to answer. No statistical association was found between town/country residency and reporting.

Conclusion* The information collected is important. It will serve to create an overview and find the best way to set up a database regarding the number of cancer patients. Taking preventive measures based on cost effective methods with the involvement of nursing and midwifery students, as a core nursing function will vastly strengthen nursing's and midwifery's contribution to gynecological cancers prevention.

\section{EFFICIENCY OF THE HYBRID CAPTURE 2 HPV DNA TEST COMPARED TO CYTOLOGY TESTING IN CERVICAL CANCER SCREENING}

${ }^{1}$ I Aluloski*, 'M Tanturovski, 'S Stojcevski, ${ }^{1} \mathrm{~V}$ Jovanovska, ${ }^{2} \mathrm{~S}$ Arsova. ${ }^{1}$ University Clinic for Obstetrics and Gynecology-Skopje, Gynecologic Oncology, Skopje, Macedonia; ${ }^{2}$ University Clinic for Obstetrics and Gynecology-Skopje, Skopje, Macedonia

\subsection{6/ijgc-2021-ESG0.563}

Introduction/Background* The aim of this study was to determine the efficiency of the Hybrid Capture 2 (HC2; Digene, Qiagen, Hilden Germany) human papillomavirus (HPV) assay for the detection of cervical neoplasia compared to conventional cytology testing.

Methodology Two hundred consecutive eligible women evaluated in the Outpatient department at the University Clinic of Gynecology and Obstetrics in Skopje aged 30-64 years, were informed and offered to be sampled with the $\mathrm{HC} 2$ test in addition to any tests ordered by their attending physician free of charge and strictly on voluntary basis. Written consent was obtained from all participating women. The attending physicians were blinded for the results of the HC2 tests and any and all medical decisions regarding patient evaluation/treatment were based on the cytology results.

Result(s)* The average age of the women included was 44 \pm 11 years, ranging from 30 to 64 . A total of 200 cytology smears, 200 HC2 tests, 76 (38\%) colposcopy examinations, 45 PCR (22.5\%) based HPV tests and 50 (25\%) cervical biopsies were performed. The detection rate for histology verified CIN2 + lesions for the HC2 based algorithm in the studied population, when compared with the conventional cytology-based algorithm was $19 / 22$ (86.36\%), while the detection rate for histology verified CIN3 + lesions was 14/ $14(100 \%)$. The sensitivity, specificity, LR + and LR- of the $\mathrm{HC} 2$ test in the detection of CIN2+ lesions were $88 \%$, $87.5 \%, 7.04$ and 0.13 , respectively, giving the test an accuracy of $87.72 \%$. Using the conventional cytology-based algorithm required 76 referrals for colposcopy, 45 referrals for PCR based HPV detection and 50 referrals for cervical biopsy, compared to 41 cytology test, 33 colposcopy referrals and 22 biopsy referrals.

Conclusion* Implementing a $\mathrm{HC} 2$ based cervical cancer screening algorithm could potentially reduce the number of colposcopy/biopsy referrals by over $50 \%$ without a substantial impact on the CIN2+ detection rate and no impact on the $\mathrm{CIN} 3+$ detection rate. This in turn decreases the associated costs of screening, decreases the number of unnecessary diagnostic procedures and, could increases patient comfort, satisfaction and adherence to the screening program 